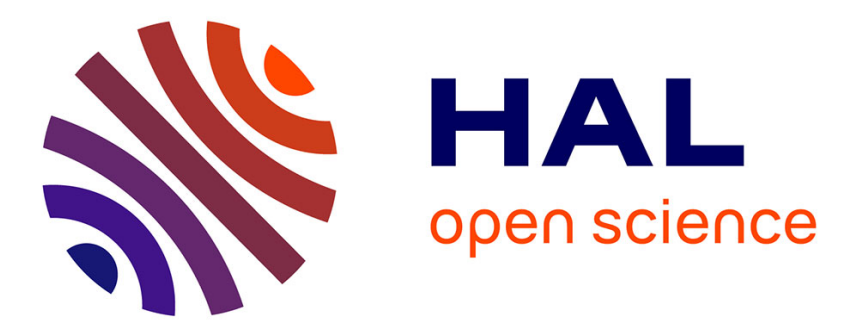

\title{
Investigation of the chromatographic process via pulsed-gradient spin-echo nuclear magnetic resonance. Role of the solvent composition in partitioning chromatography
}

\author{
Guilhem Pages, Corinne Delaurent, Stefano Caldarelli
}

\section{To cite this version:}

Guilhem Pages, Corinne Delaurent, Stefano Caldarelli. Investigation of the chromatographic process via pulsed-gradient spin-echo nuclear magnetic resonance. Role of the solvent composition in partitioning chromatography. Analytical Chemistry, 2006, 78 (2), pp.561-566. 10.1021/ac051454n . hal-02665659

\section{HAL Id: hal-02665659 \\ https://hal.inrae.fr/hal-02665659}

Submitted on 31 May 2020

HAL is a multi-disciplinary open access archive for the deposit and dissemination of scientific research documents, whether they are published or not. The documents may come from teaching and research institutions in France or abroad, or from public or private research centers.
L'archive ouverte pluridisciplinaire HAL, est destinée au dépôt et à la diffusion de documents scientifiques de niveau recherche, publiés ou non, émanant des établissements d'enseignement et de recherche français ou étrangers, des laboratoires publics ou privés. 


\title{
Investigation of the Chromatographic Process via Pulsed-Gradient Spin-Echo Nuclear Magnetic Resonance. Role of the Solvent Composition in Partitioning Chromatography
}

\author{
Guilhem Pages,* Corinne Delaurent, and Stefano Caldarelli \\ JE 2421 TRACES, Universités de Provence et Paul Cézanne Aix-Marseille I et III, Site de Saint Jérôme, \\ Case 512, Avenue Escadrille Normandie-Niemen, 13397 Marseille Cedex 20, France
}

The diffusional properties of molecules in solution vary dramatically upon addition of a solid chromatographic phase. This effect can be monitored via pulsed-gradient spin-echo NMR used in conjunction with moderately fast rotation of the sample (high-resolution magic angle spinning) to produce exploitable spectra. The molecular diffusion coefficients observed in this condition are averages reflecting the equilibrium population distribution among the different phases. It is thus possible to use this information for investigating a crucial step of reversedphase chromatography, namely, the partitioning of the analyte between different phases. In this work, we describe the evolution of the apparent diffusion coefficient of typical solutes for water/acetonitrile solvent mixtures of varying proportions.

Octadecylsilyl (ODS) bonded silica is the most frequently used chromatographic support for routine analysis in reversed-phase liquid chromatography (RPLC). The technological relevance notwithstanding, the complexity of the separation mechanism still eludes a complete description, and so NMR, among other techniques, has long been applied to investigate chromatography-related issues. Solid-state NMR has been used since the beginning of the 1990 s to characterize chromatographic phases, using ${ }^{13} \mathrm{C}$ and ${ }^{29} \mathrm{Si}$ cross-polarization/magic angle spinning NMR spectroscopy. ${ }^{1-3}$ This technique yields important information on the support structure. A major inconvenience of this approach is that it is not realized under the same conditions as LC (i.e., without solvent or compounds). Therefore, solid-state NMR can only give information about the functional groups present on the phase, but does not provide any understanding on the chromatographic phenomenon. Over the past few years, two different NMR methods have tackled this aspect. The first one is magnetic resonance imaging, ${ }^{4,5}$ which allows the study of systems under

* To whom correspondence should be addressed. E-mail: guilhem.pages@univ.u-3mrs.fr. Phone: (+33) 491288 643. Fax: (+33) 491282 897.

(1) Pesek, J. J.; Matyska, M. T.; Williamsen, E. J.; Tam, R.; Wang, Z. J. Liq. Chromatogr. Relat. Technol. 1998, 21, 2747-2762.

(2) Pesek, J. J.; Matyska, M. T.; Yu, R. J. J. Chromatogr., A 2002, 947, 195203.

(3) Pursch, M.; Sander, L. C.; Albert, K. Anal. Chem. 1999, 71, 733A-741A.

(4) Tallarek, U.; Baumeister, E.; Albert, K.; Bayer, E.; Guiochon, G. J. Chromatogr., A 1995, 696, 1-18.

10.1021/ac051454n CCC: $\$ 33.50$ (C) 2006 American Chemical Society Published on Web 12/06/2005 real chromatographic conditions in the presence of a flow of a liquid and in a packed column, but in this case, the addition of a contrast agent (mainly gadolinium chelates) is necessary to record good contrasted images. This technique has never been used to study typical solutes of liquid chromatography. The second method is high-resolution magic angle spinning (HRMAS) $\mathrm{NMR}^{6-9}$ by which classical mixtures could be analyzed under static conditions (that is with no flow of the mobile phase).

The tool used in this work combines diffusion-oriented NMR and HRMAS. By NMR, it is possible to measure apparent diffusion coefficient (ADC) using pulsed gradients of magnetic field in conjunction with spin-echos (PGSE). ${ }^{10,11}$ A recent proposal in this field by Caldarelli and co-workers ${ }^{12}$ accomplished an enhancement of the resolution in the diffusion dimension using a solid phase to create specific molecular interactions. The varying degree of decrease in the molecular mobility due to interactions with the solid eventually leads to resolution of the NMR spectra of a mixture components. The addition of the support compels the use of the standard NMR technique for solids, MAS, to remove the anisotropic parts of the interactions and so to achieve a NMR spectrum with narrow lines. The overall process can be seen as an extension of the so-called "affinity NMR". Variations of the ADC have been used, for example, to investigate peptide-micelle association, ${ }^{13}$ ligands-receptor interactions, ${ }^{14}$ or for screening new potential chromatographic phases. ${ }^{15}$

(5) Boughtflower, B. J.; Mutton, I. M.; Paterson, C. J.; Reid, D. G.; Busza, A. L.; Blackaby, A. P. Analyst 2004, 129, 124-129.

(6) Baumeister, E.; Klose, U.; Albert, K.; Bayer, E.; Guiochon, G. J. Chromatogr., A 1995, 694, 321-331.

(7) Brindle, R.; Pursch, M.; Albert, K. Solid State Nucl. Magn. Reson. 1996, 6, 251-266.

(8) Händel, H.; Gesele, E.; Gottschall, K.; Albert, K. Angew. Chem., Int. Ed. 2003, 42, 438-442.

(9) Hellriegel, C.; Skogsberg, U.; Albert, K.; Lämmerhofer, M.; Maier, N. M.; Lindner, W. J. Am. Chem. Soc. 2004, 126, 3809-3816.

(10) Stejskal, E. O.; Tanner, J. E. J. Chem. Phys. 1965, 42, 288-292.

(11) Stilbs, P. Prog. Nucl. Magn. Reson. Spectrosc. 1987, 19, 1-45.

(12) Viel, S.; Ziarelli, F.; Caldarelli, S. Proc. Natl. Acad. Sci. U.S.A. 2003, 100, 9696-9698.

(13) Deaton, K. R.; Feyen, E. A.; Nkulabi, H. J.; Morris, K. F. Magn. Reson. Chem. 2001, 39, 276-282.

(14) Gounarides, J. S.; Chen, A.; Shapiro, M. J. J. Chromatogr., B 1999, 725, 79-90.

(15) Laverde, A., Jr.; Da Conceicao, G. J. A.; Queiroz, S. C. N.; Fujiwara, F. Y.; Marsaioli, A. J. Magn. Reson. Chem. 2002, 40, 433-442. 


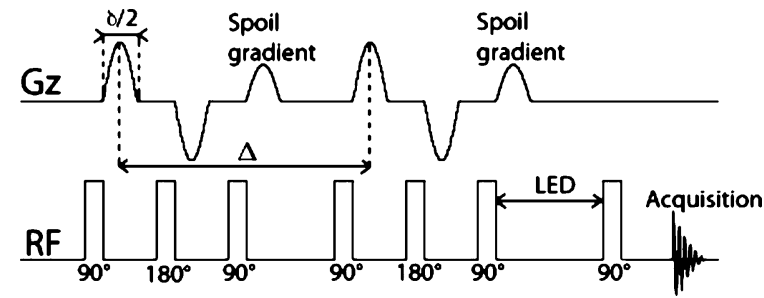

Figure 1. BPP-LED pulse sequence. The picture shows the radio frequency (rf) and gradient $(\mathrm{Gz})$ pulses.

\section{Table 1. Diffusion Parameters Used during the} BPP-LED Sequences ${ }^{a}$

\begin{tabular}{lccc} 
& & \multicolumn{2}{c}{ with phase } \\
\cline { 3 - 4 }$\delta / 2(\mathrm{~ms})$ & $0.5-0.75$ & $0.7-0.8$ & aromatics \\
$\Delta(\mathrm{ms})$ & $40-60$ & $75-100$ & $75-800$ \\
& & & \\
${ }^{a} \Delta / 2$ is the gradient pulse duration, & and $\Delta$ the diffusion time.
\end{tabular}

The combination of HRMAS and PGSE NMR allows us to investigate one aspect of the chromatographic phenomenon, namely, the effect of varying the mobile-phase composition on the separation process. To this purpose, an aromatic and a ketone homologous series in a water/acetonitrile mixture were used.

\section{EXPERIMENTAL SECTION}

Materials and Methods. All NMR experiments were performed on a Bruker (Wissembourg, France) Avance spectrometer operating at $400 \mathrm{MHz}$ and equipped with a HRMAS probe head capable of producing $z$ gradients with maximum strength of $55 \mathrm{G}$ $\mathrm{cm}^{-1} .{ }^{1} \mathrm{H}$ HRMAS NMR spectra were recorded at a spinning rate of $4000 \mathrm{~Hz}$ at $303 \mathrm{~K}$. Four-millimeter $\mathrm{ZrO}_{2}$ rotor with a detection volume of $55 \mu \mathrm{L}$ were used. The free induction decay contained typically 2 and 8 or $16 \mathrm{~K}$ data points, respectively, for spectra recorded with and without chromatographic phase.

The pulse sequence (Figure 1) used was based on the stimulated echo and incorporated gradient pulses and a longitudinal eddy delay (BPP-LED). ${ }^{16}$

The 90 and $180^{\circ}$ pulses were optimized for each experiment, being about 5 and $10 \mu \mathrm{s}$, respectively. Table 1 summarizes the parameters used for NMR diffusion experiments.

The shape of all gradient pulses was sinusoidal. The spoil gradient pulses were $1 \mathrm{~ms}$ and the LED $5 \mathrm{~ms}$. A BPP-LED experiment was realized using 16 different gradient values, varying from 2 to $95 \%$ of the maximum gradient strength; for each of them, 16 scans were recorded. For the processing, 128 points were represented in the diffusion dimension.

All compounds, including HPLC solvents, were obtained from VWR (Fontenay sous Bois, France). The chromatographic phase was Lichrospher100® C18 (5 $\mu \mathrm{m})$ kindly provided by Merck (Darmsdat, Germany).

The test mixture for the aromatic homologous series were benzene $\left(9.0 \mathrm{~g} \mathrm{~L}^{-1}\right)$, naphthalene $\left(0.9 \mathrm{~g} \mathrm{~L}^{-1}\right)$, and anthracene $(0.2$ $\left.\mathrm{g} \mathrm{L}^{-1}\right)$ dissolved in different solvent compositions. The ketone (acetone, butanone, and pentan-2-one) sample contained $10 \mu \mathrm{L}$ of

(16) Wu D. H.; Chen A. D.; Johnson C. S. J. Magn. Reson., A 1995, 115, 260264. solute and $100 \mu \mathrm{L}$ of deuterated solvent varying from 100 to $60 \%$ v/v $\mathrm{CD}_{3} \mathrm{CN}$. For each experiment, the rotor contained about 35 mg of chromatographic phase and $10 \mu \mathrm{L}$ of the test mixture. Roughly half of the stationary phase was introduced inside the rotor, then the solution was added, and finally the rotor was filled with the remaining part of the solid.

Analysis of Molecular Diffusion by NMR. The inhomogeneous field created by the pulsed gradients induces a spatial dependence of the NMR frequency and, consequently, a broadening of the resonance line. This latter can be made proportional to the molecular mobility through appropriate acquisition schemes. For the BPP-LED sequence, the molecule is inferred from the following equation,

$$
I=I_{\mathrm{o}} \exp [-D(\delta g \gamma)(\Delta-\delta / 3)]
$$

where $I$ is the observed intensity of the peak, $I_{0}$ the intensity of the peak measured in the absence of field gradients, $D$ the ADC, $\delta$ the gradient pulse duration, $g$ the gradient strength, $\gamma$ the gyromagnetic ratio of the observed nucleus, and $\Delta$ the diffusion time.

The study of a series of experiments varying the amplitude of the inhomogeneous field and investigating the corresponding decay curve of the signal amplitude permits us to measure the $\mathrm{ADC}$. The DOSY processing ${ }^{17,18}$ is a representation of the signal decay curve in the form of a 2D map, in which the NMR spectrum spans one dimension and the calculated diffusion coefficient the other. The diffusion coefficients were also determined by fitting the decay curves with the manufacturer software XWinNMR 3.5.

\section{RESULTS AND DISCUSSION}

Deuterated versus Nondeuterated Solvents. It is common practice in ${ }^{1} \mathrm{H}$ NMR to use deuterated solvents to avoid nuisances from the overwhelming solvent signal. The adequateness of this choice was questioned at the time of the introduction of hyphenated HPLC NMR methods, due to the high cost of deuterated solvents. In this respect, new acquisition schemes have been developed, and it is now possible to use, with some caution, nondeuterated solvents in hyphenated methods. In the case of an analysis that intends to look into the chromatographic process, it should be kept in mind that RPLC can separate deuterated and protonated solutes. ${ }^{19,20}$ This difference in retention times between the two isotopomers is a result of variations in the intermolecular interactions between the molecules and the stationary phase. This shows that, in principle, one isotopomer cannot be used instead of the other in liquid chromatography because their retention times could be different. The same holds true for our diffusion measurements, in principle. However, this setup may not be practical when the resonance due to the test and the solvent molecules overlaps in the NMR spectrum. Thus, we directly investigated the solvent isotope influence on the observed mobility on both the phase (i.e., the flexible part of the solid phase) and some test molecules. The peaks of the chromatographic phase that are observed in the DOSY map (a part of the alkyl chains)

(17) Johnson, C. S. Prog. Nucl. Magn. Reson. Spectrosc. 1999, 34, 203-256.

(18) Morris, K. F.; Johnson, C. S., Jr. J. Am. Chem. Soc. 1992, 114, 3139-3141. (19) Shi, B.; Davis, B. H. J. Chromatogr., A 1996, 731, 351-354.

(20) Jinno, K. J. High Resolut. Chromatogr., Chromatogr. Commun. 1982, 5, 364367. 


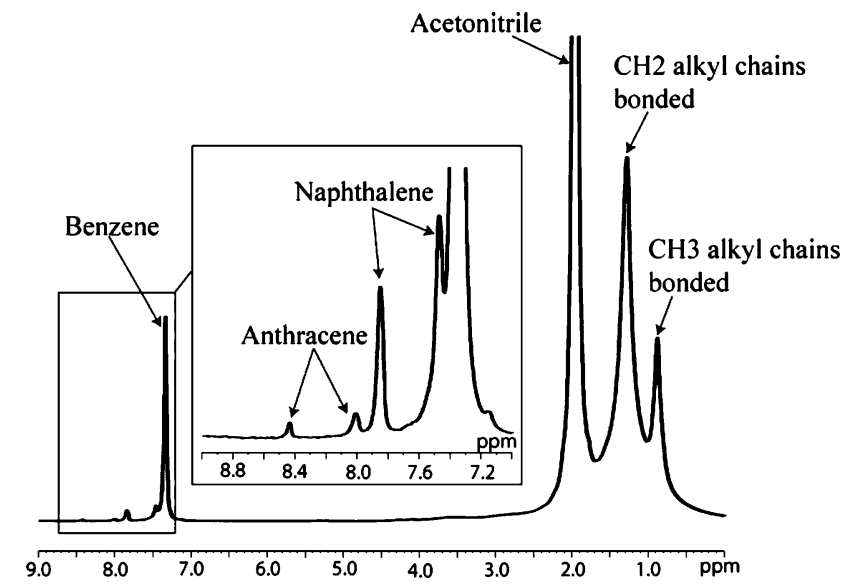

Figure 2. ${ }^{1} \mathrm{H}$ MAS NMR spectrum of a homologous aromatic series in pure acetonitrile with the octadecylsilyl bonded silica gel inside the rotor.

show a biexponential decay along the diffusion dimension. In the case of deuterated acetonitrile as a solvent, the two diffusion components were $(1.2 \pm 0.1) \times 10^{-11}$ and $(2.3 \pm 0.5) \times 10^{-10} \mathrm{~m}^{2}$ $\mathrm{s}^{-1}$. With a protonated HPLC grade solvent, we obtained a diffusion coefficient of about 8 times faster $\left((9.5 \pm 0.1) \times 10^{-11}\right.$ $\left.\mathrm{m}^{2} \mathrm{~s}^{-1}\right)$ for the slow component and 16 times faster $((3.7 \pm 0.5)$ $\left.\times 10^{-9} \mathrm{~m}^{2} \mathrm{~s}^{-1}\right)$ for the fast component. This suggests that alkyl chains bonded on silica gel do not have the same organization with deuterated or protonated solvent. In the case of the aromatic homologous series, the analytes had the same ADC in deuterated or protonated solvent, suggesting that the different chromatographic phase organization entailed by the isotopomer had no influence on the observed equilibrate and, also, on the diffusion measured. It can be concluded that the different isotopomers do not influence the NMR observables in our experiment.

In the following analysis on homologous series, we used protonated solvents in the case of the aromatic molecules. The same was not possible for the ketone series because an overlap between the acetonitrile signal (1.94 ppm) and the ketone methyl signal $(2.15 \mathrm{ppm})$ is observed, due to the limited resolution caused by the presence of chromatographic phase, and deuterated solvents were employed.
NMR Mobilities in Homologous Series Varying Solvent Mixtures. Homologous series commonly used in chromatography for estimations of column performances in terms of both efficiency and selectivity include aromatic molecules, $n$-alcohol, $n$-alkylbenzene, and $n$-ketone. We used an aromatic (benzene, naphthalene, and anthracene) and a ketone (acetone, butanone, and pentan-2one) series to perform our study.

$\mathrm{A}^{1} \mathrm{H}$ MAS NMR spectrum of the ODS bonded phase with the mixture of aromatic solutes in acetonitrile is presented in Figure 2.

The resolution enhancement in the diffusion NMR induced by the addition of a chromatographic phase is pointed out in Figures 3 and 4. Without the chromatographic phase, the diffusion dimension exhibits a poor resolution (Figures $3 \mathrm{a}$ and $4 \mathrm{a}$ ), meaning not appreciable differences in the mobility of the set molecules. With the support, ADCs differences become a factor of 35 between benzene and naphthalene and 7 between naphthalene and anthracene (Figures $3 \mathrm{~b}$ and $4 \mathrm{~b}$ ), greatly increasing the resolution in the diffusion dimension. Even under magic angle spinning, the resolution recovered in the first dimension (the NMR chemical shift) is weaker than for the corresponding spectra in a pure solution, due to residual anisotropic effects..$^{21}$ Overall, the mobility variation between aromatic solutes does not exceed $6 \%$ in the absence of the solid support. Instead, the use of the ODS chromatographic phase gives a maximum relative variation of $100 \%$ between the slowest and fastest compound, providing an evident separation of the spectra of the molecular systems in the diffusion dimension.

A similar behavior is observed for the ketone series (Figure 5 ), with poor resolution of the molecular spectra without the chromatographic support (Figure 5a). The butanone and pentan2-one are essentially indistinguishable from their diffusional properties. The addition of the solid phase (Figure $5 \mathrm{~b}$ ) doubles the resolution between these two molecules. The less effective $\mathrm{ADC}$ separation of the ketone ensemble with respect to the aromatic ones (Figures $3 \mathrm{~b}$ and $5 \mathrm{~b}$ ) parallels the scale of selectivity observed in HPLC, as in our interpretation, the delay observed in the $\mathrm{ADC}$ is directly related to the residence time. Ketones are less retained than aromatic molecules in these conditions.
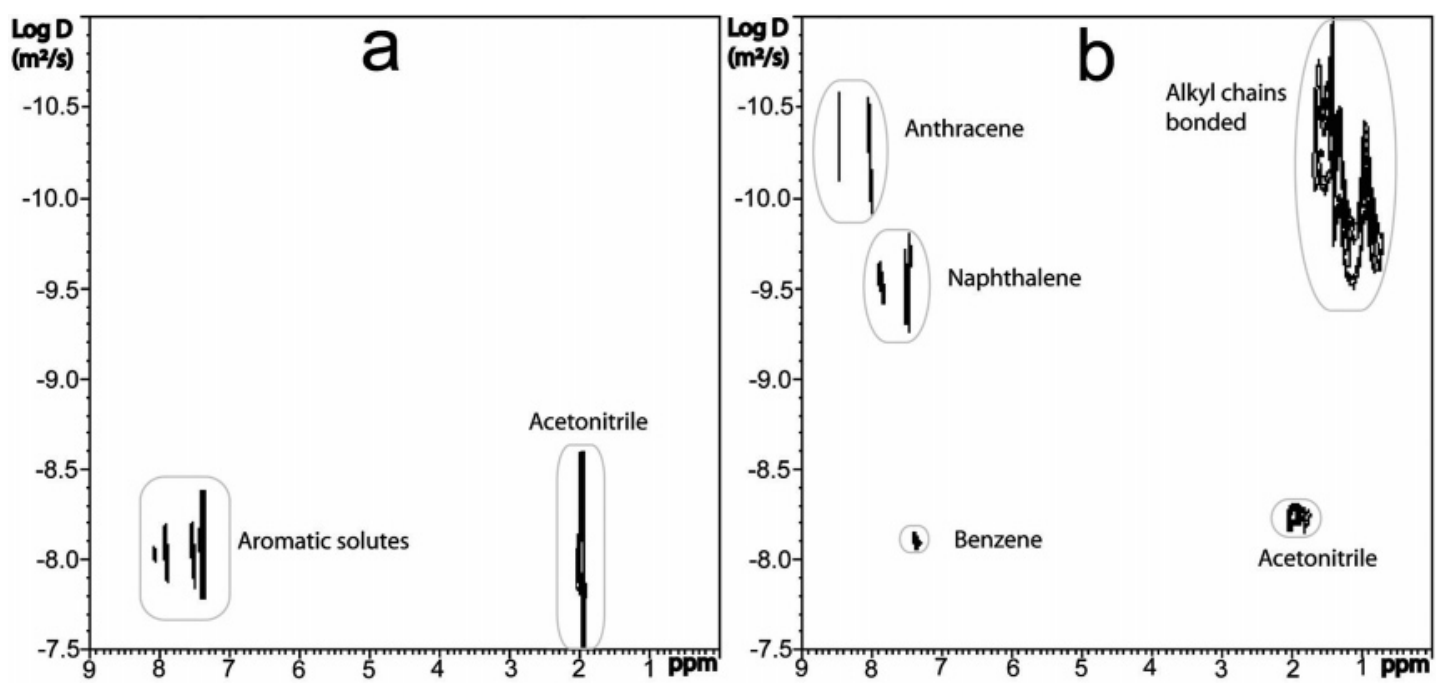

Figure 3. ${ }^{1} \mathrm{H}$ DOSY NMR spectra of a homologous aromatic series in pure acetonitrile (a) without and (b) with the presence of the chromatographic support. 

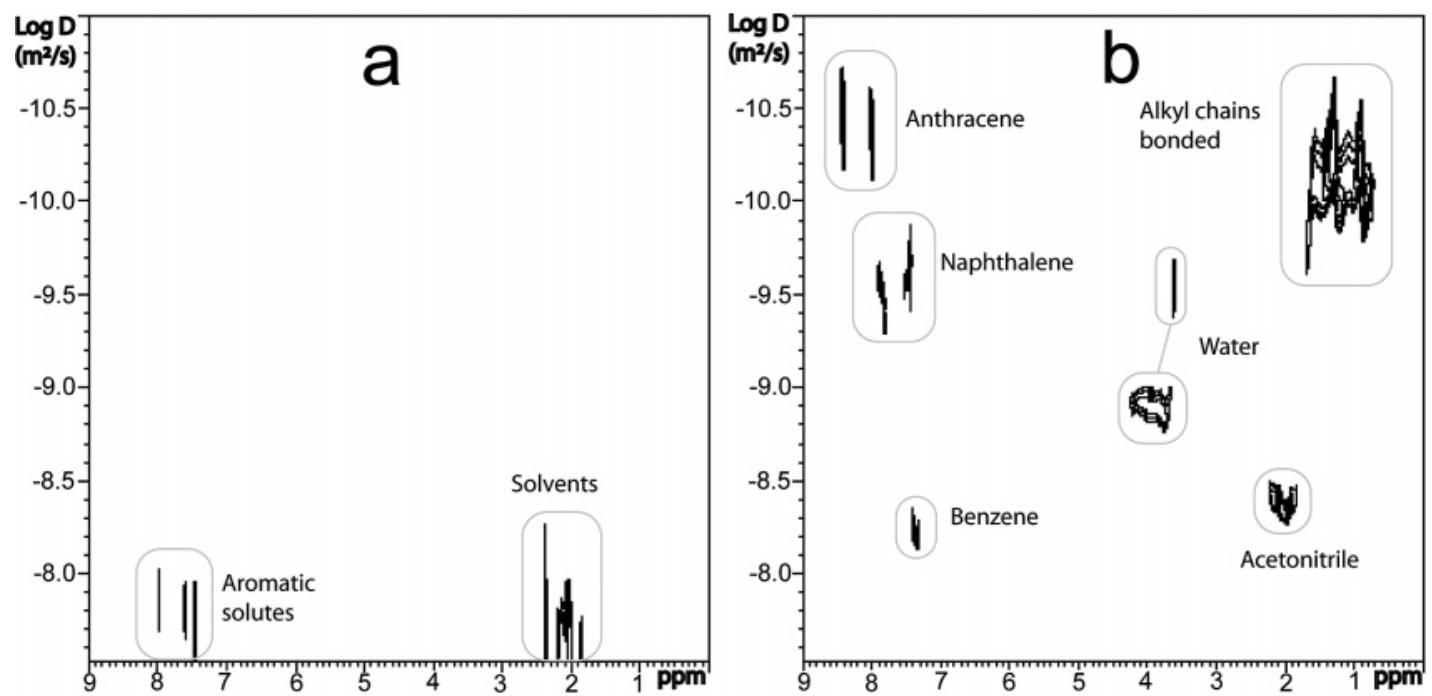

Figure 4. ${ }^{1} \mathrm{H}$ DOSY NMR spectra of a homologous aromatic series in a acetonitrile/water mixture $(80 / 20$, v/v) (a) without and (b) with the presence of the chromatographic support.
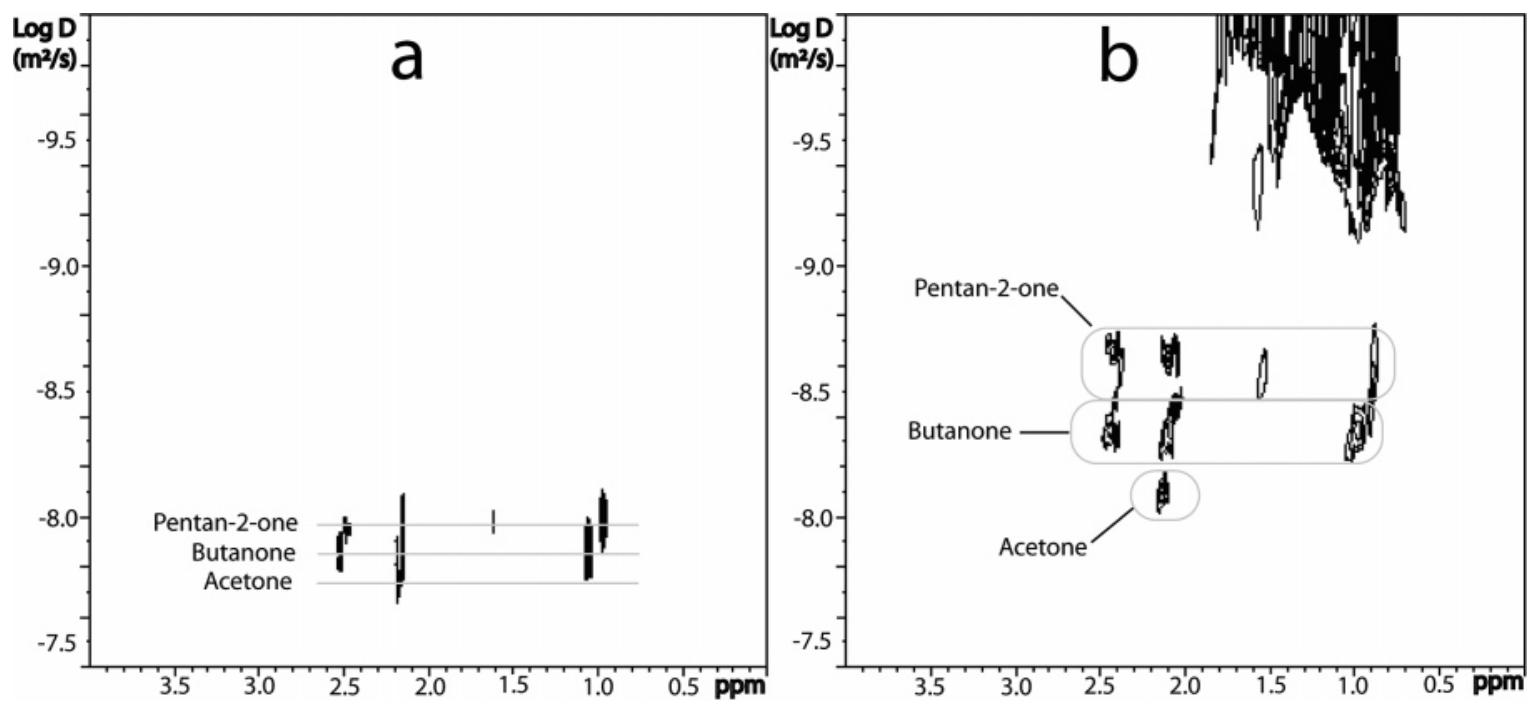

Figure 5. ${ }^{1} \mathrm{H}$ DOSY NMR spectra of a homologous ketone series in acetonitrile- $d_{3}$ (a) without and (b) with the addition of ODS silica gel. Spectra are the sum of the ones of the three individual molecules, recorded separately.

The resolution observed in the diffusion dimension of these DOSY charts (Figures $3-5$ ), due to the intrinsic line widths, is reduced in the presence of a solid with respect to experiments performed on solutions. While the processing of this kind of data can still be considered in its infancy and not completely appropriate to provide the best resolution, ${ }^{22}$ there may be some physical explanations for such a line broadening. In fact, the observed average process that gives rise to the NMR peak can still suffer from anisotropic effects, namely, variations of the local environment or of the diffusion properties for a subset of molecules. At the extreme, all of the above could translate into peak splitting and to a line broadening on a milder scale. Experiments are underway to investigate these particular aspects.

Role of the Mobile-Phase Composition. In liquid-phase chromatography, the mobile-phase composition has a strong influence on the separation quality and selectivity. The effect of using, as a liquid phase, a water/acetonitrile mixture was thus investigated next. Optimized ratios of the two liquids are known to be necessary for optimal separation of a given analyte mixture.
We varied the solvent composition from 70 to $100 \%$ and from 60 to $100 \%$ in acetonitrile for the aromatic and ketone series, respectively, since for this latter series higher water content are expected for optimal resolution.

Incidentally, Figure $4 \mathrm{~b}$, representing the aromatic series in a solvent containing $20 \%$ water in the presence of chromatographic phase, shows that the water signal is associated with two different $\mathrm{ADCs}$. The first value of the $\mathrm{ADC}$ corresponds roughly to the one of free water $\left((2.3 \pm 0.3) \times 10^{-9} \mathrm{~m}^{2} \mathrm{~s}^{-1}\right)$, while the second one is $\sim 7$ times slower $\left((3.1 \pm 0.1) \times 10^{-10} \mathrm{~m}^{2} \mathrm{~s}^{-1}\right)$. This difference in $\mathrm{ADC}$ is of the same order as the value measured by Lindon and coauthors $^{23}$ and confirms the existence of two different populations of water: the first one is close to free water, and the second one may correspond to water trapped inside ODS bonded silica pores.

(21) Morcombe, C. R.; Zilm, K. W. J. Magn. Reson. 2003, 162, 479-486.

(22) Thureau, P.; Thévand, A.; Ancian, B.; Escavabaja, P.; Armstrong, G. S.; Mandelshtam, V. A. ChemPhysChem 2005, 6, 1510-1513.

(23) Coen, M.; Wilson, I. D.; Nicholson, J. K.; Tang, H.; Lindon, J. C. Anal. Chem. 2004, 76, 3023-3028. 

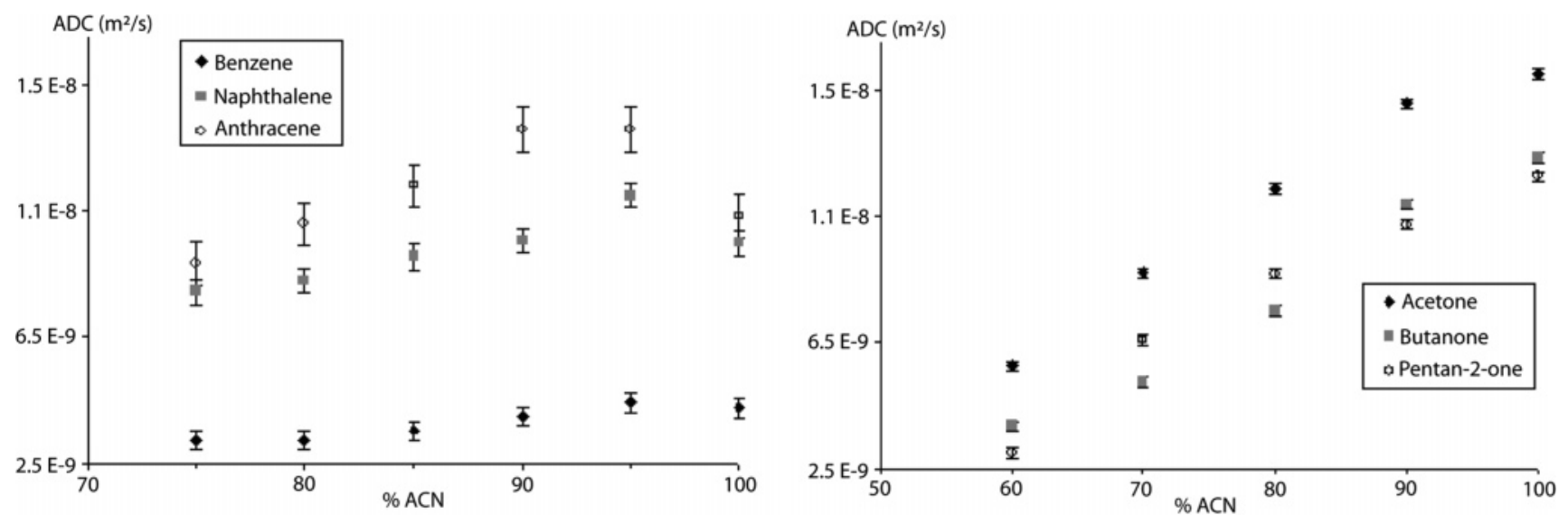

Figure 6. Evolution of the ADC for the both homologous series as a function of the solvent composition without chromatographic support. On the left panel the aromatic set, and on the right panel the ketone one.
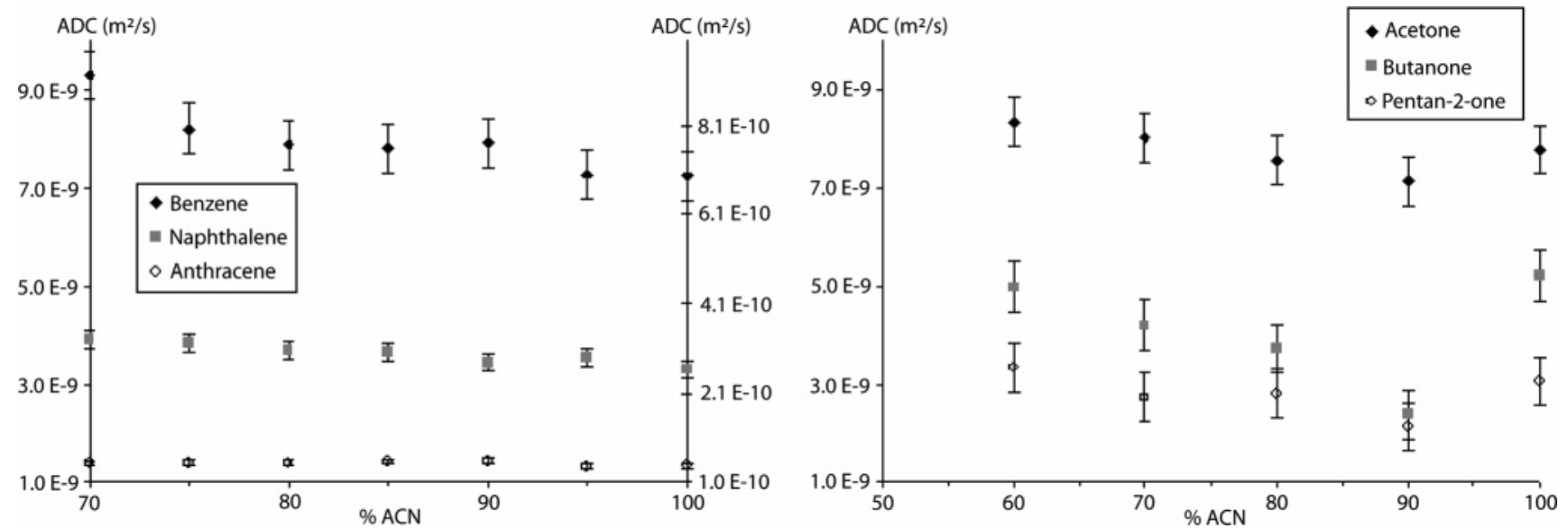

Figure 7. Evolution of the ADC for the both homologous series as a function of the solvent composition in the presence of the chromatographic support. Left panel: aromatic series, with the scale relative to benzene on the right-hand side and the one relative to the two other molecules on the left-hand side. Right panel: ketone series.

For all liquid-phase compositions, the mobile-based separation quality observed by NMR was again weaker for the ketone series than for aromatic solutes as observed in liquid chromatography.

Since the effect detected by our technique is the weighed average of molecular diffusion over all possible phases, we first measured the incidence of varying the solvent composition on the mobilities of the test molecules in the pure liquid. In fact, this is the only state of the matter in the final mixture that can be easily described. Without the chromatographic phase, all ADC are lower for higher water content (Figure 6) roughly by a factor from 1.5 to 4.0 over the range tested, consistently with the increasing viscosity of the solution. The opposite trend is observed in the presence of the ODS silica gel. In this case, the evolution of the molecular diffusion rates is the opposite and the ADC of the solutes increases when water is added (Figure 7).

To clarify further the comparison between our results and chromatography, we built an equivalent of a chromatogram by reporting the inverse of the $\mathrm{ADC}$ as a fictitious retention time (Figures 8 and 9). The less retained solute is therefore on the left of the figure and the most retained is on the right.

In this "NMR retention time" scale, the solute with fastest mobility would correspond to the first eluted one. In Figure 8, the order of "NMR elution" is equivalent to the chromatographic one. For the aromatic series, the first eluted solute is benzene,

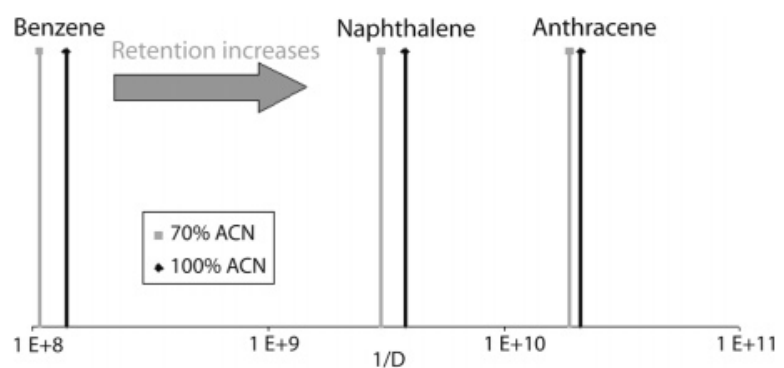

Figure 8. Illustration of the ADC evolution of the aromatic homologous series for two values of the solvent composition in the form of a chromatogram. The retention is expressed as the inverse of the ADC, and the vertical scale is fixed.

then naphthalene, and finally anthracene. In this representation, the difference in "retention time" between a solvent composition with 0 and $30 \%$ of water shows that the addition of this latter has more influence on benzene than on naphthalene and little effect on anthracene mobilities. A similar trend is observed for butanone and pentan-2-one in Figure 9, where the impact of the water addition is also inversely proportional to the molecular size. This is not the case for acetone, which is commonly used for the determination of void volume in RPLC. However, RPLC experimental evidence shows that acetone is at least weakly retained under all mobile-phase compositions and that retention increases 


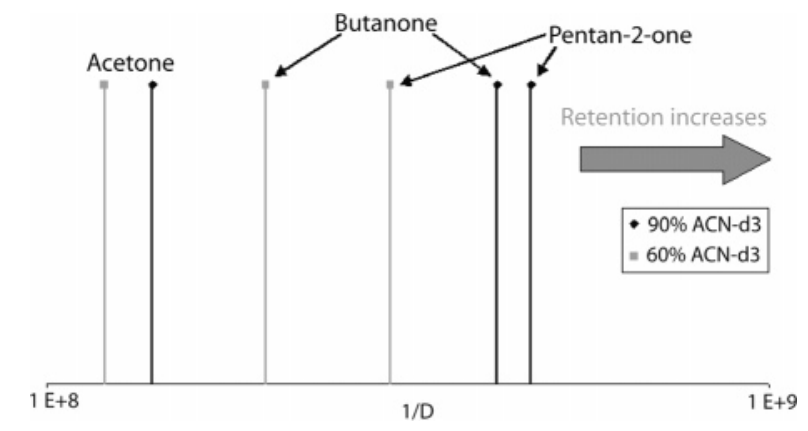

Figure 9. Same representation as in Figure 8 for the ketone series.

with the water content. These considerations are consistent with the weak influence of water on the acetone ADC.

The effect of the solvent composition on the separation selectivity, defined as the resolving power of the method, in the case of the NMR analysis parallels the known trend of HPLC. On the other hand, the solute mobility is inversely proportional to the water content in the liquid phase (i.e., the NMR retention time decreases). This is contrary to the commonly observed behavior of RPLC, where the retention time increases when water is added to the mobile phase. This difference cannot be easily explained at this stage but may be due to the fact that we only observe a part of the chromatographic process. The mixture resulting from the addition of a liquid and a chromatographic stationary phase can be seen as a combination of intermediate phases, with respect to the different mobilities. A probe molecule, such as the test ones used in our homologous series, explores the different environments as a function of their accessibility. Namely, the addition of water induces the unfolding of the grafted organic chains, thus unblanking the access to a part of the porous surface while at the same time providing a new semimobile environment. A part of the water is retained by the chromatographic phase, as testified by the two diffusion rates observed for the water peak. Coincidently, the $\mathrm{ADC}$ of acetonitrile also decreases when water is added, providing further evidence that addition of water increases the interaction of the liquid phase with the silica gel. Similarly, the different solute molecules will populate the various layers according to the relative affinities and size. A qualitative idea of the upper limit of the mobility of these layers can be found in the most mobile-phase peaks in the DOSY display, corresponding to the terminal bent of the chains.

(24) Howerton, S. B.; McGuffin, V. L. J. Chromatogr., A 2004, 1030, 3-12.

(25) Hong, L.; Felinger, A.; Kaczmarski, K.; Guiochon, G. Chem. Eng. Sci. 2004, 59, 3399-3412.
For instance, anthracene has the slowest average mobilities, being even less mobile than alkyl bonded chains, as this molecule likely penetrates deeper in the stationary phase ${ }^{24}$ than other probe solutes. Within this representation of the average mobility, the trend observed for a given molecule while changing the mobilephase composition must be explained either by a variation in the population of the different layers or by changes in the diffusion rate typical of each layer. For example, Guiochon and coauthors ${ }^{25}$ showed that, for the different diffusions measured in RPLC, only the intraparticle diffusion increases with the water percentage in the mobile phase.

\section{CONCLUSIONS}

We provided an analysis of the evolution of ADC of small molecules dissolved in a liquid in the presence of ODS silica. HRMAS NMR provides the resolution necessary to distinguish relevant details of the molecular motion.

The analysis can be performed in deuterated solvents without appreciable effect on the partition of the analytes among the different phases present in the sample.

The variation of the liquid-phase composition, following typical cases employed in HPLC, is reflected by the mobility of the mixture components. Particularly, high water concentration in a mixture with acetonitrile amplifies the difference in mobilities in typical test sets of homologous molecules, a result consistent with the increased selectivity observed in HPLC for the same conditions. However, a striking difference with liquid chromatography is observed as this higher selectivity is associated usually with longer retention times, while in our experiments an acceleration of the molecular mobility is found.

We are designing some experiments to further describe this behavior.

\section{ACKNOWLEDGMENT}

This work was supported by the French Conseil Régional Provence-Alpes-Côte-d'Azur. The authors are also grateful for financial support received from the Pharmaceutical Laboratory DISTRI B3 (Marseille, France).

Received for review August 12, 2005. Accepted November 7, 2005.

AC051454N 